\title{
Wearable Bluetooth Low Energy Based Miniaturized Detection Node for Blind Spot Detection and Warning System on Vehicles
}

\author{
$1^{\text {st }}$ Nick De Raeve \\ Dept. of Information Technology \\ Ghent University - imec, IDLab \\ Ghent, Belgium \\ nick.deraeve@ugent.be
}

\author{
$4^{\text {th }}$ Jo Verhaevert \\ Dept. of Information Technology \\ Ghent University - imec, IDLab \\ Ghent, Belgium \\ jo.verhaevert@ugent.be
}

\author{
$2^{\text {nd }}$ Quinten Van den Brande \\ Dept. of Information Technology \\ Ghent University - imec, IDLab \\ Ghent, Belgium \\ quinten.vandenbrande@ugent.be
}

\author{
$3^{\text {rd }}$ Matthias de Schepper \\ Dept. of Information Technology \\ Ghent University - imec, IDLab \\ Ghent, Belgium \\ matthias.deschepper@ugent.be
}

\author{
$5^{\text {th }}$ Patrick Van Torre \\ Dept. of Information Technology \\ Ghent University - imec, IDLab \\ Ghent, Belgium \\ patrick.vantorre@ugent.be \\ $6^{\text {th }}$ Hendrik Rogier \\ Dept of Information Technology \\ Ghent University - imec, IDLab \\ Ghent, Belgium \\ hendrik.rogier@ugent.be
}

\begin{abstract}
Annually, approximately 10 people are involved in a lethal blind spot accident on Belgian roads, even though a lot of money is invested in the development of blind spot detection systems and in raising the awareness of this phenomenon. In previous research, we developed a blind spot detection and warning system based on Bluetooth Low Energy (BLE) and received signal strength indicator (RSSI) measurements. In this paper, the miniaturization of the detection node and wearable is presented. There will be a closer look at the development of the Printed Circuit Board (PCB) and the folded Shorted Patch (S-P) antenna that will be integrated into the side lights of trailers. In a future step, the wearable design will be updated with the same miniaturization steps taken in this paper.
\end{abstract}

Index Terms-BLE, Bluetooth Low Energy, Blind spot detection

\section{INTRODUCTION}

In 2020, approximately 100 people were killed on Belgium roads [1]. $10 \%$ of the killed are due to a blind spot accident. Annually, a lot of money is invested by government and traffic safety organizations to raise awareness of this problem. However, the danger still exists. The main problem lies in the lack of reliable communication between the truck driver and the vulnerable road user. To eliminate or possibly solve this problem, we proposed a blind spot detection and warning system based on Bluetooth Low Energy (BLE) in a previous work [2]. In this paper, there will be a closer look at the miniaturization of the detection node, so it can be fitted inside the small lights alongside a truck trailer.

Industries provide multiple solutions, each with their disadvantages [3], [4]. In literature, multiple research is conducted in the placing of cameras [5] and the optimization of different algorithm types [6], [7] to detect vulnerable road users in the blind spot. It is a known fact that camera systems suffer from some known problems. Most of them have a limited coverage area, have a bad performance when dirty and are hindered by privacy regulations. Combined with a high implementation cost, cameras are not the most ideal solution. Furthermore, some research is performed in detection based on radar [8]. Both systems will detect vulnerable road users, but suffer from several false positives and true negatives [3], [4]. Besides camera and radar, there are also the systems that use wearables [2], [9]. These utilize an on-body wearable to detect how close they are to a truck. Once too close they will trigger an alert so the vulnerable road user and truck driver are notified about a potential blind spot accident.

This paper presents the miniaturization of the detection node. First, there will be a closer look at the design of the Printed Circuit Board (PCB) and the folded Shorted Patch (S$\mathrm{P})$ antenna. It is followed by the measurements and discussion of the PCB and the antenna. Finally, the conclusion and future work are presented.

\section{DESIGN}

The proposed system from [2] consists of five detection nodes placed alongside a truck with a trailer. A central node is positioned inside the cabin of the truck. The detection nodes are continuously advertising the presence of the truck. A vulnerable road user is wearing the wearable, also proposed in [2] and can be seen in Figure 1, and receives all these advertisement packages. Based on the received signal strength indicator (RSSI) values, the possibility of an accident is calculated. When both parties are too close, an alert is triggered on the wearable device, but the wearable also connects to the closest detection node. Based on this connection, another alert is triggered in the cabin of the truck. From this moment, there is a shared responsibility between both parties and important lifesaving safety measures can be taken. The wearable worn 
by the vulnerable road user contains leds, buzzer and vibration motor to alarm the person in every possible method.

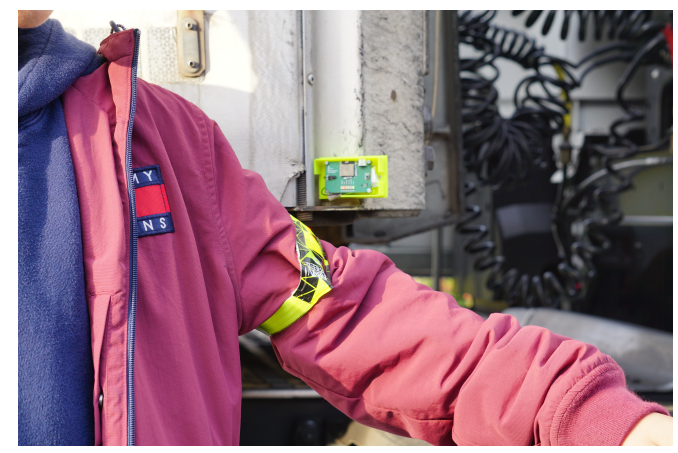

Fig. 1: Student wearing the designed wearable next to a truck, that is equipped with the designed system.

The communication between the different nodes of the proposed system relies on BLE. Therefore, we utilize the BGM111 module from Silicon Laboratories [10] is utilized. These modules use the BLE 4.2 stack [11] and contain an onboard 32-bit, 38.4 MHz ARM Cortex M4 [12] Microcontroller (MCU) with DSP instruction set, combined with an integrated antenna. The PCB of the detection node, integrated in an inhouse constructed plastic 3D-printed box, is shown in Figure 2. Besides the electronic components, this PCB contains also the chip antenna and has a size of $45 \times 30 \mathrm{~mm}$, necessary as ground plane for the chip antenna.

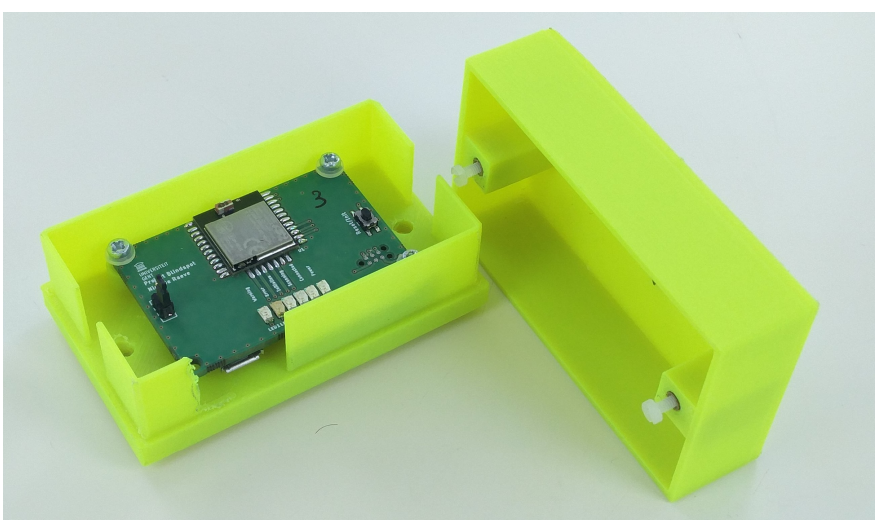

Fig. 2: Designed detection node presented in [2].

This system is upgraded by a miniaturization of the detection node. Due to the required ground plane size for the chip antenna, these detection nodes cannot be built inside a side light of a trailer, as can be seen in Figure 3. Therefore, a miniaturization of the detection node is required, taking into account the following constraints. First of all, the PCB has to fit inside the housing of the side light, resulting in dimensions smaller than $30 \times 20 \mathrm{~mm}$. Further, the module in the detection has to run on BLE Mesh [13] to facilitate more convenient data transfer between the detection nodes and the central node. Finally, since the node will be implemented inside the metal chassis of the trailer, a dedicated and adapted antenna has to be developed, satisfying the aforementioned dimension restrictions.

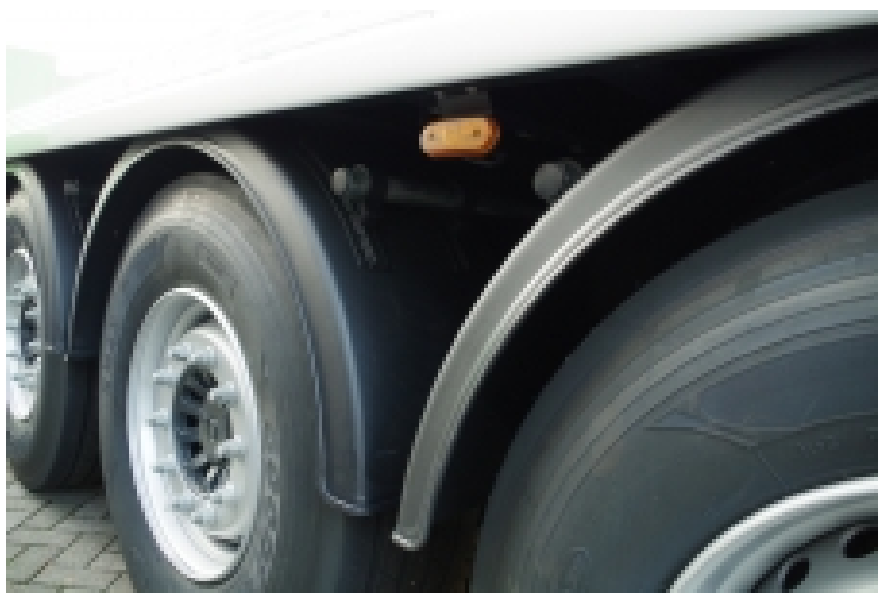

Fig. 3: Picture of a side light on European Truck.

\section{A. Printed Circuit Board}

The design of the miniaturized PCB relies on the Silicon Laboratories EFR32MG13 [14] System-on-Chip (SOC). It contains a 32-bit ARM [12] Cortex-M4 core with $40 \mathrm{MHz}$ maximum operating frequency, $512 \mathrm{kB}$ of flash and $64 \mathrm{kB}$ of RAM, an integrated power amplifier with up to $19 \mathrm{dBm}$ and an integrated balun for $2.4 \mathrm{GHz}$. Furthermore, this SOC also supports the use of BLE Mesh [13]. This all is combined in a package of $5 \times 5 \mathrm{~mm}$, which results in a serious downsize compared to the earlier mentioned BGM111.

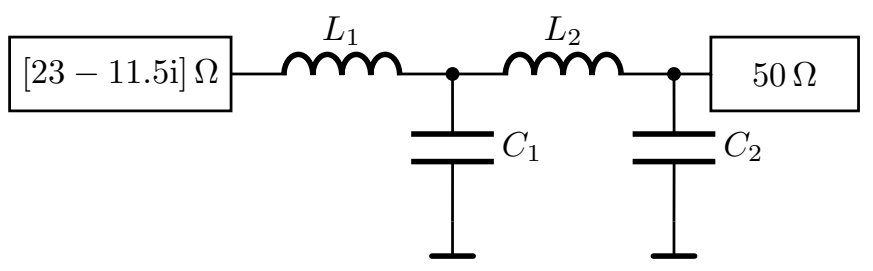

Fig. 4: Applied matching network.

An SMA connector has been added to the PCB for easier testing of multiple antenna topologies. To provide maximal power transfer towards the antenna, a fourth order LCmatching network is designed in order to conjugate match the $50 \Omega$ SMA connector interface to the $[23-11.5 \mathrm{i}] \Omega$ impedance of the EFR32MG13P MCU, as can be seen in Figure 4. The matching network, described further, determines the values for $\mathrm{L}$ and $\mathrm{C}$, as is given in Table I. Furthermore, by implementing Grounded Co-Planar Waveguide (GCPW) [15], the traces are minimized even further. Taking all these steps into account,

TABLE I: Calculated LC values.

\begin{tabular}{|c|c||c|c|}
\hline Inductor & Value [nH] & Capacitor & Value [pF] \\
\hline \hline$L_{1}$ & 1.3 & $C_{1}$ & 5.3 \\
\hline$L_{2}$ & 1.5 & $C_{2}$ & 3.2 \\
\hline
\end{tabular}




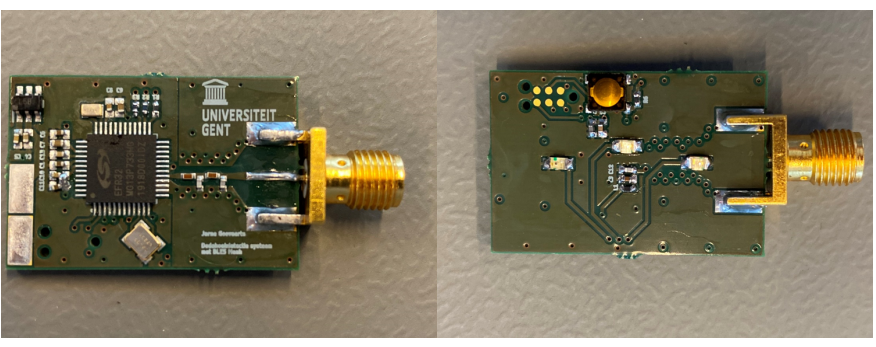

Fig. 5: Designed miniaturized PCB for the detection node.

a new PCB is designed and is visualized in Figure 5. This PCB has an overall size of $30 \times 20 \mathrm{~mm}$, which is a serious reduction in size compared with the previous design.

\section{B. Miniaturized BLE antenna}

As the antenna is required to support the IEEE 802.15.1 standard (BLE mesh), a reflection coefficient with respect to $50 \Omega,\left|S_{11}\right|$, below $-10 \mathrm{~dB}$ is imposed in the $[2.400$ $2.4835] \mathrm{GHz}$ frequency band. In addition a 3 dB-beamwidth greater than $70^{\circ}$ in the azimuth-plane is imposed in order to ensure sufficient coverage in a large area next to the truck. Finally, maximum dimensions for the antenna are set at $35 \times 25 \mathrm{~mm}$ to fit the antenna inside the side light of the truck. Note that special care is required when integrating the antenna inside the light, as the large metallic structures can severely influence the antenna performance.

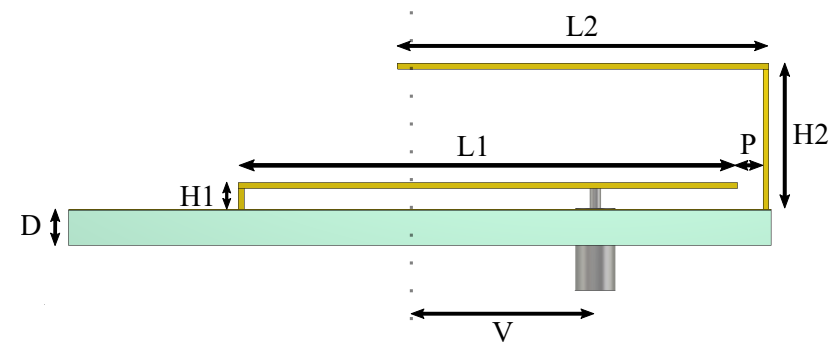

(a) yz-plane

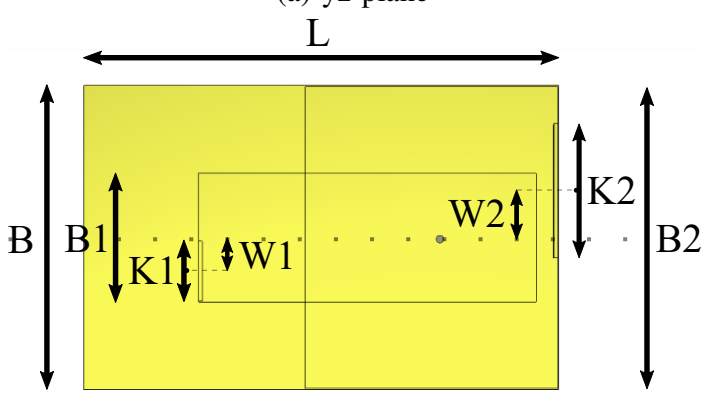

(b) xy-plane

Fig. 6: Annotated representation of the folded Shorted-Patch (S-P) antenna constructed out of $0.25 \mathrm{~mm}$ brass sheets.

To realize the imposed requirements a folded Shorted-Patch antenna, based on [16], is proposed. The antenna essentially
TABLE II: Dimensions after optimization of the folded Shorted-Patch (S-P) antenna.

\begin{tabular}{|c|c||c|c|}
\hline Dimension & Value $[\mathrm{mm}]$ & Dimension & Value [mm] \\
\hline \hline$L$ & 31.26 & $L 2$ & 16.53 \\
\hline$B$ & 20.00 & $B 2$ & 19.72 \\
\hline$D$ & 1.60 & $H 2$ & 6.50 \\
\hline$L 1$ & 22.20 & $W 2$ & 3.06 \\
\hline$B 1$ & 8.45 & $K 2$ & 8.80 \\
\hline$H 1$ & 1.19 & $P$ & 1.39 \\
\hline$W 1$ & 2.20 & $V$ & 4.77 \\
\hline$K 1$ & 3.94 & & \\
\hline
\end{tabular}

consists of two planar inverted-F antennas (PIFA) implemented on an air substrate constructed out of $0.25 \mathrm{~mm}$ brass sheets, as depicted also in Figure 6. By stacking both PIFA elements on top of each other and, hence, creating a tight coupling between them, mode bifurcation is achieved [17]. By judiciously optimizing the respective resonance frequencies of the antenna, a strong miniaturization of the antenna structure is obtained, meanwhile taking into account the above described design criteria. The final antenna dimensions after optimization are depicted in Table II.

\section{Simulations \& DISCUSSION}

After designing the PCB, the matching network as well as the proposed antenna topology is simulated.

\section{A. Matching network}

In subsection II-A, the fourth order LC-matching network is discussed. It is used to match the MCU with the SMA connector. GCPWs further minimize the necessary PCB traces. The values for the matching can be found in Table I, whereas all the different sizes of the waveguide can be extracted from Figure 8 .

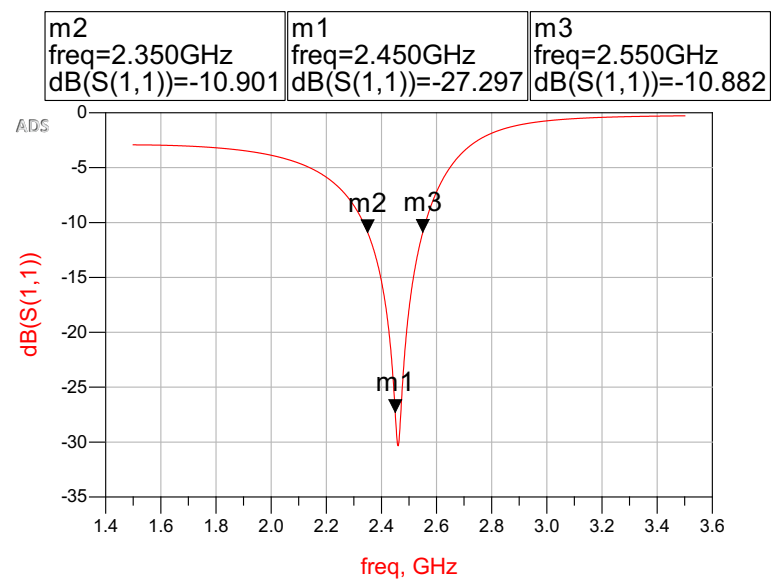

Fig. 7: Simulated $S_{11}$ of the designed matching network.

The $S_{11}$ of the designed matching network is visualized in Figure 7. At the center frequency of $2.45 \mathrm{GHz}$, there is a $S_{11}$ of $-27.3 \mathrm{~dB}$. The $S_{11}$ is smaller than $-10 \mathrm{~dB}$ in the entire frequency band [2.34-2.56] GHz. This results in an impedance bandwidth of $\pm 200 \mathrm{MHz}$. 


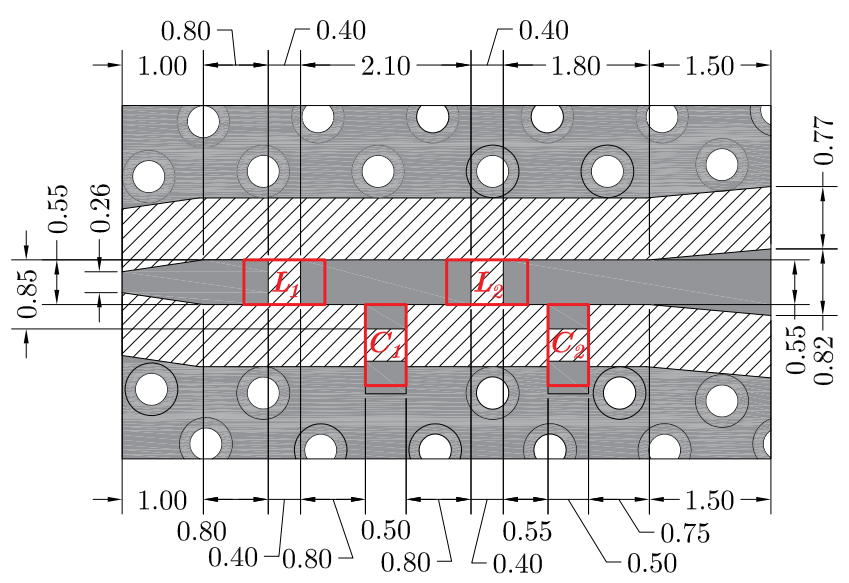

Fig. 8: Designed matching network with implemented GCPW.

\section{B. Folded Shorted-Patch antenna results}

The simulated S-parameters of the optimized antenna, depicted in Figure 10, show a $\left|S_{11}\right|<-10 \mathrm{~dB}$ from $2.35 \mathrm{GHz}$ to $2.55 \mathrm{GHz}$. The simulated gain pattern in the azimuth and elevation plane is shown in Figure 11a and Figure 11b, showing a peak gain of $2.1 \mathrm{dBi}$ and $2.4 \mathrm{dBi}$, respectively. A $3 \mathrm{~dB}$-beamwidth of $360^{\circ}$ and $103.6^{\circ}$ can be observed in the azimuth and elevation plane, respectively.

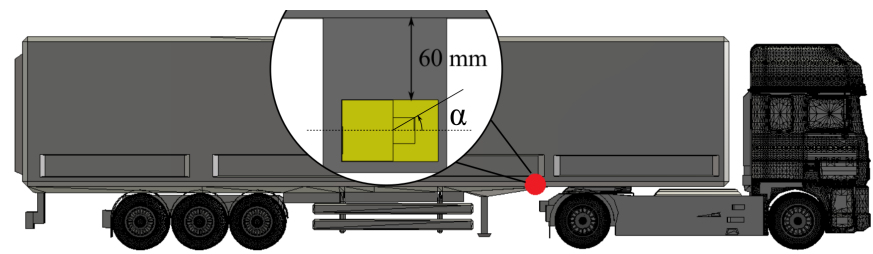

Fig. 9: Truck with antenna integration orientation $\alpha=0^{\circ}$.

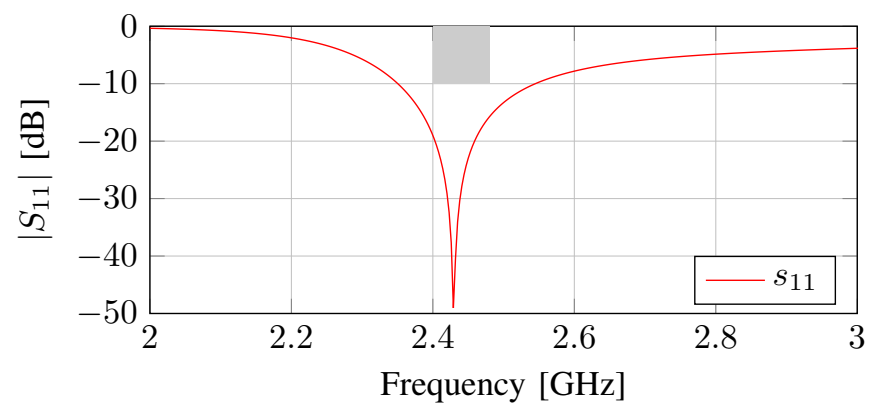

Fig. 10: Simulated $S_{11}$ of folded S-P antenna constructed out of $0.25 \mathrm{~mm}$ brass sheets.

In order to validate the antenna performance in realistic deployment scenarios, the folded S-P antenna is also simulated when integrated into a side light on the trailer of a truck, as depicted in Figure 9. Here, the red dot indicates the location of the antenna on the trailer, behind the last wheel of the truck.

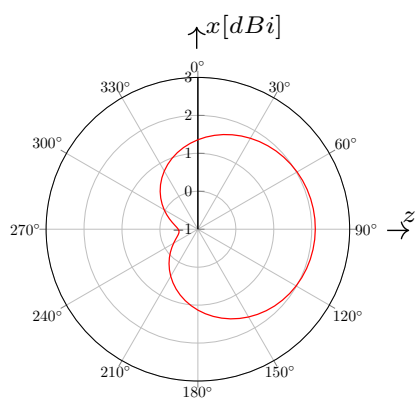

(a) Azimuth.

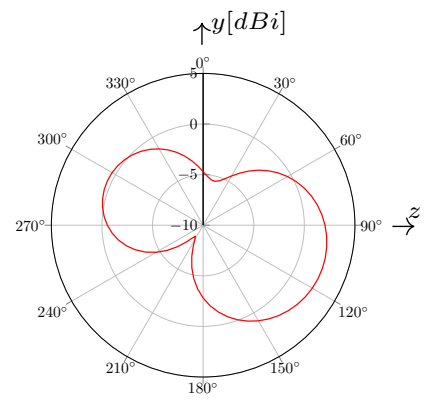

(b) Elevation.
Fig. 11: Simulated gain of the standalone folded S-P antenna constructed out of $0.25 \mathrm{~mm}$ brass sheets.

The antenna is placed $60 \mathrm{~mm}$ below the container frame of the trailer.

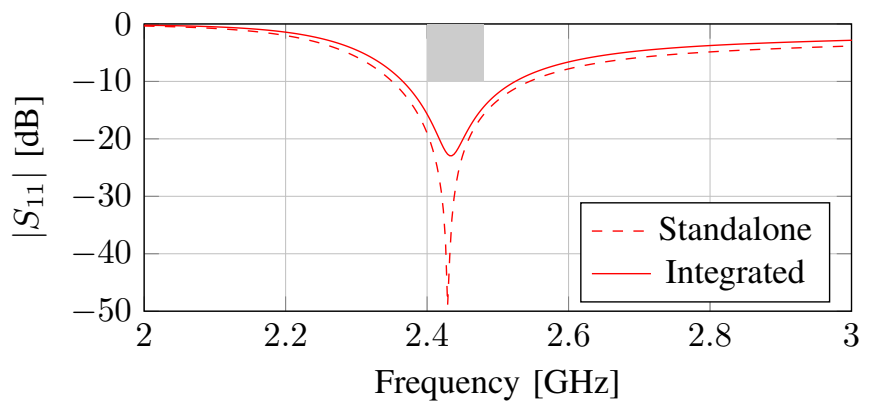

Fig. 12: Simulated $S_{11}$ of folded S-P antenna constructed out of $0.25 \mathrm{~mm}$ brass sheets integrated on a truck.

Figure 12 shows that the effect of the integration platform is negligible on the antenna impedance and, as such, on the reflection coefficient. To analyze the impact of the truck on the radiation performance, the radiation pattern is simulated for different antenna integration orientations, defined by the angle $\alpha$. Again, the integration platform has negligible influence on the radiation pattern when $\alpha=0^{\circ}$ or $\alpha=180^{\circ}$, as can be seen in Figure 13. However in contrast, when $\alpha=90^{\circ}$ the integration platform lowers the main beam direction in the elevation platform, thereby decreasing the overall gain in the azimuth plane.

\section{CONCLUSION}

This paper describes the upgrading and miniaturization of a previously developed detection node for blind spot detection and warning system for vehicles based on BLE. The entire system consists of five detection nodes placed alongside a truck, continuously transmitting advertising packets to potential wearable nodes in its vicinity. In order to integrate the detection nodes into the trailer side lights, a miniaturized and highly integrated design is proposed.

To this end, both the PCB and antenna have been redesigned: a smaller PCB footprint is obtained and a miniaturized folded S-P antenna is designed to fit within this 


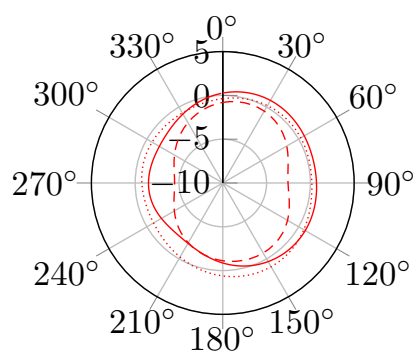

(a) Azimuth

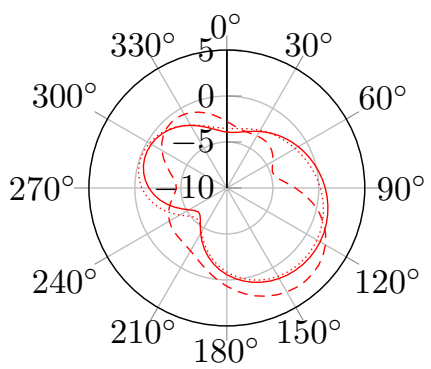

(b) Elevation
Fig. 13: Simulated gain of the integrated folded S-P antenna constructed out of $0.25 \mathrm{~mm}$ brass sheets for different integration orientations. The solid line represents $\alpha=0^{\circ}$, the dashed line $\alpha=90^{\circ}$ and the dotted line $\alpha=180^{\circ}$.

footprint. A fourth order matching network is added to convert the transceiver output impedance from $[23-11.5 \mathrm{i}] \Omega$ to the required $50 \Omega$. All these measures result in a perfect integrable design.

\section{FUTURE WORK}

In a future work, there will be more optimizations of the entire system. For the detection node, the following measures will be implemented. First of all, to decrease the overall size, the antenna will be matched to the impedance of the MCU. In that way, the matching network can be reduced and both the PCB and the antenna can be integrated on one PCB.

Further, by implementing BLE Mesh [13], it will be more convenient to transfer data between different detection nodes, but also towards the central node. Replacing a detection node will be cheaper and less complicated due to the provisioning process [18] of BLE Mesh.

Finally, by implementing Angle of Arrival (AOA) [19], the system will be able to provide more accurate detection.

By utilizing the same SOC and PCB design, the wearable can be reduced in size as well. This will also improve the possibility to redevelop the solid PCB wearable to a flex or flex-rigid design, making the wearable more unobtrusive. Additionally, the PCB could be integrated in other equipment that is used for walking, cycling or daily carrying.

\section{REFERENCES}

[1] VIAS, "Road safety barometer," https://www.vias.be/en/research/roadsafety-monitoring-survey/, accessed on September 9, 2021.

[2] N. De Raeve, M. de Schepper, J. Verhaevert, P. Van Torre, and H. Rogier, "A bluetooth-low-energy-based detection and warning system for vulnerable road users in the blind spot of vehicles," Sensors, vol. 20, no. 9, 2020.

[3] VIAS, "Accidents involving Trucks - Phase 1," https://www.vias.be/en/onderzoek/onze-publicaties/ongevallen-metvrachtwagens-fase-1/, accessed on September 9, 2021.

[4] — , "In-depth investigation of crashes involving heavy goods vehicles," https://www.vias.be/en/onderzoek/onze-publicaties/ongevallenmet-vrachtwagens-fase-2/, accessed on September 9, 2021.

[5] N. Stojanovic, I. Grujic, J. Glisovic, O. I. Abdullah, and S. Vasiljevic, "Application of new technologies to improve the visual field of heavy duty vehicles' drivers," in New Technologies, Development and Application II. Springer International Publishing, Apr. 2019, pp. 411-421.
[6] H. Lee, M. Ra, and W.-Y. Kim, "Nighttime data augmentation using gan for improving blind-spot detection," IEEE Access, vol. 8, pp. 48049 $48059,2020$.

[7] D. Kwon, R. Malaiya, G. Yoon, J.-T. Ryu, and S.-Y. Pi, "A study on development of the camera-based blind spot detection system using the deep learning methodology," Applied Sciences, vol. 9, no. 14, 2019. [Online]. Available: https://www.mdpi.com/2076-3417/9/14/2941

[8] G. Liu, L. Wang, and S. Zou, "A radar-based blind spot detection and warning system for driver assistance," in 2017 IEEE Second Advanced Information Technology, Electronic and Automation Control Conference (IAEAC), March 2017, pp. 2204-2208.

[9] J. Verhaevert, "Detection of vulnerable road users in blind spots through bluetooth low energy," in 2017 PROGRESS IN ELECTROMAGNETICS RESEARCH SYMPOSIUM - SPRING (PIERS), 2017, pp. 227-231.

[10] Silicon Laboratories, "BGM111 Blue Gecko Bluetooth module data sheet," https://www.silabs.com/documents/public/data-sheets, accessed on September 9, 2021.

[11] Bluetooth Special Interest Group, "Specification of the Bluetooth System, covered core package version 4.2," https://www.bluetooth.com/specifications/archived-specifications/, accessed on September 9, 2021.

[12] ARM, https://www.arm.com/products/silicon-ip-cpu, accessed on September 9, 2021.

[13] Bluetooth Special Interest Group, "Mesh networking is blue," https://www.bluetooth.com/learn-about-bluetooth/recentenhancements/mesh/, accessed on September 9, 2021.

[14] Silicon Laboratories, "EFR32MG13 Series 1 Modules," https://www.silabs.com/wireless/zigbee/efr32mg13-series-1-modules, accessed on September 9, 2021.

[15] M. Bozzi, A. Georgiadis, and K. Wu, "Review of substrate-integrated waveguide circuits and antennas," Microwaves, Antennas \& Propagation, IET, vol. 5, pp. 909 - 920, 072011.

[16] R. Li, G. DeJean, M. Tentzeris, and J. Laskar, "Development and analysis of a folded shorted-patch antenna with reduced size," IEEE Transactions on Antennas and Propagation, vol. 52, no. 2, pp. 555562, 2004.

[17] Q. Van den Brande, S. Lemey, J. Vanfleteren, and H. Rogier, "Highly efficient impulse-radio ultra-wideband cavity-backed slot antenna in stacked air-filled substrate integrated waveguide technology," IEEE Transactions on Antennas and Propagation, vol. 66, no. 5, pp. 21992209, 2018.

[18] Bluetooth Special Interest Group, "Provisioning a Bluetooth Mesh Network," https://www.bluetooth.com/blog/provisioning-a-bluetooth-meshnetwork-part-1/, accessed on September 9, 2021.

[19] - "How AoA \& AoD Changed the Direction of Bluetooth Location Services," https://www.bluetooth.com/blog/new-aoa-aod-bluetoothcapabilities/, accessed on September 9, 2021. 